Full-field interferometry using infinity corrected optics

This content has been downloaded from IOPscience. Please scroll down to see the full text. 2016 Meas. Sci. Technol. 27015402

(http://iopscience.iop.org/0957-0233/27/1/015402)

View the table of contents for this issue, or go to the journal homepage for more

Download details:

IP Address: 138.250.83.56

This content was downloaded on 01/12/2015 at 09:51

Please note that terms and conditions apply. 


\title{
Full-field interferometry using infinity corrected optics
}

\author{
T O H Charrett and R P Tatam \\ Engineering Photonics, Cranfield University, MK43 0AL, UK \\ E-mail: t.charrett@cranfield.ac.uk
}

Received 28 August 2015, revised 28 September 2015

Accepted for publication 16 October 2015

Published 1 December 2015

\begin{abstract}
In this paper the construction of full-field (imaging) interferometers using infinity corrected optics commonly used in microscopy is discussed, with an emphasis on self-mixing interferometry configurations where the imaged light field is mixed with itself rather than a reference wave. Such configurations are used in speckle shearing interferometry, flow visualisation and quantitative flow measurement. The critical considerations for constructing path-length imbalanced full-field interferometers for these and similar applications are discussed, expressions are derived for key calculations and interferograms from example interferometers are presented. These include the concept of balancing the infinity-spaces of the two arms via the use of a glass block to minimise the optical path difference variation across the interferogram and ensure adequate sampling of the fringes on the detector. Further, the use of tilted glass blocks in single-pass and double-pass arrangements is detailed for the generation and control of spatial carrier fringes without extensive realignment of the interferometer, and for phase shifting.
\end{abstract}

Keywords: infinity corrected optical systems, interferometric imaging, fringe analysis, shearography, planar Doppler velocimetry (PDV), Doppler global velocimetry (DGV)

(Some figures may appear in colour only in the online journal)

\section{Introduction}

Infinity corrected optics are commonly used in microscope systems and offer high quality imaging together with a flexible space between objective and tube lens, shown in figure 1, termed the 'infinity-space', for the mounting of additional optical components [1].

However the use of infinity corrected microscope optics in full-field interferometry instrumentation is mainly limited to the use of special interferometry objectives, in Michelson and Mirau geometries, or the use of matched objective lenses in Linnik interferometers [2] for non-contact optical profile measurement and surface measurements. These interferometry objectives have a built-in beam splitter to divide the illumination and provide a reference, and as such they do not allow self-mixing configurations where the light from the object plane is mixed with itself. For example, such selfmixing configurations have been used in areas such as flow visualisation [3] and quantitative flow measurements [4-7], where the illumination is provided in the form of a laser light sheet and a path length imbalanced interferometer is used as an optical filter to resolve Doppler frequency shifts, and in speckle shearing interferometry [8-10] where the speckle pattern from an illuminated test object is mixed with an offset version of itself. In both of these applications the light collected from the object plane is divided into the two arms of the interferometer before being recombined and the resulting interference pattern recorded. This is in contrast to interferometry objectives where the light from the object plane is interfered with reference light taken from the illumination source.

Self-mixing, imaging interferometers for these applications can however be constructed using standard infinity-corrected microscope objectives $[4,5,11,12]$. This offers the advantages of high imaging quality provided by off-the-shelf microscope components, and a flexible imaging system in which the imaging optics can be easily switched depending upon 


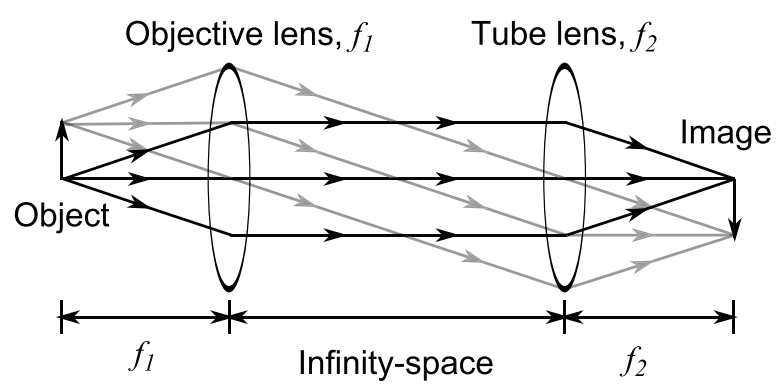

Figure 1. Basic schematic of an infinity corrected optical system.

the application requirements without major reconfiguration of the interferometer itself. Such a set-up can be used; with a single imaging lens $[4,11]$, for endoscopic measurements [12], or to port multiple images from imaging fibre bundles allowing spatially multiplexed measurements [5]. As such the use of infinity-corrected optical systems have great potential in full-field interferometry for many applications in optical instrumentation, including vibration and strain measurement $[9,10]$, flow field visualisations [3] and quantitative velocity measurements [4-7].

However this approach is not common, possibly due to the differences between constructing a full-field interferometer with a conventional imaging set-up and using an infinitycorrected design. This paper addresses some of the critical considerations for constructing full-field interferometers utilising infinity-corrected optics for these and other applications. An overview of the basic principles of infinity corrected imaging systems is first given in section 2 . This is then followed by details of the critical concept of balancing the effective infinity space of the interferometer for path length imbalanced interferometers in section 3. Without this balancing, the optical path difference across the image will vary too rapidly leading to under-sampling of the resulting fringes away from the optical axis. Finally useful practical implementations for the introduction of linear carrier fringes, as required for spatial carrier phase evaluation methods [13, 14] and for phase shifting using the tilt of a glass block introduced into one arm of the interferometer are presented in section 4 and section 5 .

\section{Infinity-corrected imaging systems}

The basic principle of an infinity-corrected optical system is shown in figure 1, where all light from a point in the object plane, located at the focal length of the first/objective lens, passes through the intermediate space between the two lenses as collimated bundles of rays. This so-called infinity space is typically $165-200 \mathrm{~mm}$ [1] for microscope systems, although the length of this space is flexible and can be adjusted. In practice the upper limit will depend upon the acceptable level of vignetting, due to outer rays in the bundles being lost to the system. Only light from a point on the optical axis will pass through parallel to the optical axis, all light from other locations in the object plane, $x$, will be at some angle, $\theta$, to the optical axis that depends upon the focal length of the first lens/ objective lens, $f_{1}$, and is given by: a)

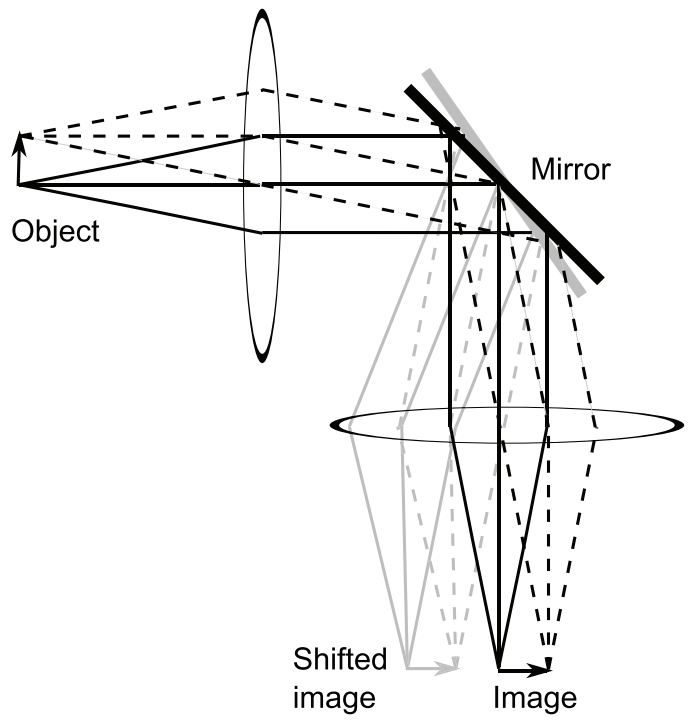

b)

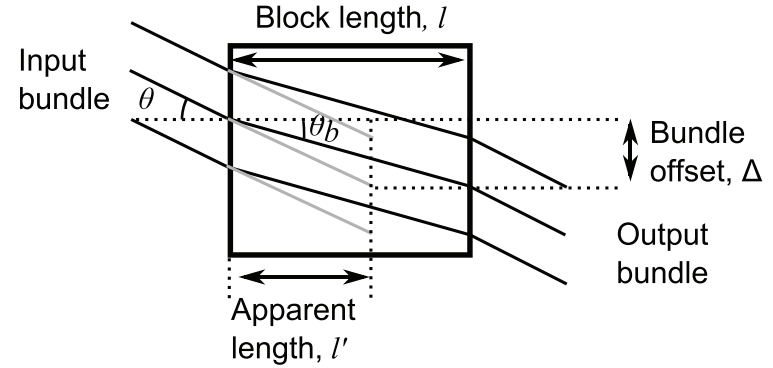

Figure 2. Illustrating (a) the effect of placing a tilted mirror and (b) a glass block; inside the infinity-space. In (b) it can be seen that a glass block will result in a smaller bundle offset, hence the apparent infinity space length of the block is less than that for an equivalent air path.

$$
\theta=\tan ^{-1}\left(x / f_{1}\right)
$$

The position in the image plane, $x^{\prime}$ can be determined from the bundle angle and the focal length of the second lens/tube lens, $f_{2}$ and substituting equation (1):

$$
x^{\prime}=-f_{2} \tan \theta=M x
$$

Where the magnification, $M$, of such a system is given by:

$$
M=-f_{2} / f_{1}
$$

The addition of optical components inside the infinity-space can thus have two influences; the first is to change the bundle angle, $\theta$, and hence the image position, while the second is to change the optical path length travelled by the light from a particular position in the object plane. For example a tilted mirror, figure 2(a), will result in a change to the bundle angle that is constant for all bundle angles, resulting in a translated image, while a tilted glass block, figure 2(b), will have no effect on the image position, as the bundle angle leaving the block is the same as that entering the block.

However in the second case there will be an increase in the optical path length due to the higher refractive index of the block and the bundle offset from the optical axis will be reduced leading to reduced optical losses from vignetting as the extreme rays from higher angle bundles will no longer 
be lost from the system, figure 2(b). Such a glass block can also be said to provide an apparent infinity-space length, which is equivalent to the shorter length of air giving the same bundle offset, even though the optical path length is actually increased. From geometry and Snell's law the apparent infinity-space length, $l_{b}^{\prime}$, of a glass block, with a length of $l_{b}$ can be shown to be:

$$
l_{b}^{\prime}(\theta)=\frac{\tan \left(\theta_{b}\right)}{\tan (\theta)} \cdot l_{b}=\sqrt{\frac{\left(1-\sin ^{2} \theta\right)}{\left(n_{g} / n_{a}\right)^{2}-\sin ^{2} \theta}} \cdot l_{b}
$$

Where $\theta, \theta_{b}, n_{a}$ and $n_{g}$, are the bundle angles and refractive indices in air and glass block respectively. As the values of $\theta$ are typically small this can then be estimated at $\theta=0$ giving:

$$
l_{b}^{\prime}(\theta)=\left(\frac{n_{a}}{n_{g}}\right) \cdot l_{b}
$$

Other components, such as beam splitters and right-angle prisms, can be thought of as a combination of these reflection and refraction effects.

\section{Minimising the optical path difference variation across the image}

A critical consideration when constructing a full-field pathlength imbalanced interferometer using infinity-corrected optics is to ensure that the variation of the optical path difference (OPD) across the image is minimised. This variation is due to the fact that the light travels through the infinity space at different angles to the optical axis depending upon the position in the object/image planes and hence the optical path-length will also vary with bundle angle/image position. For path-length imbalanced interferometers, this leads to a varying OPD across the image resulting in bull's-eye fringes in which the fringes will have a rapidly increasing frequency with radial distance.

If the OPD variation is not minimised, then for practical applications such as interferometric planar Doppler velocimetry $[4,11]$ that require large path length differences $(\sim 360 \mathrm{~mm})$ between the two arms, the OPD will vary too rapidly across the image, hence the fringes will not be adequately sampled by the detector and phase evaluation will not be possible. It is therefore necessary to reduce the variation of the OPD across the image in some way. This can be achieved by using a glass block in one arm of the interferometer. This block will not only add optical path length to that arm, but will also reduce the effective infinity-space length, due to the reduced bundle angle inside the block, and reduce the light losses due to vignetting. Due to the dependency of the bundle angles on the object/image position the optical path in each arm will still vary across the image, however as the bundle angles in the first arm are reduced due to refraction at the block interface it is possible to set the air path in the second arm to approximately 'balance' this change of path-length, and provide a near constant OPD across the image.

The air path necessary to achieve this balancing can be estimated using expressions for the path-lengths present in
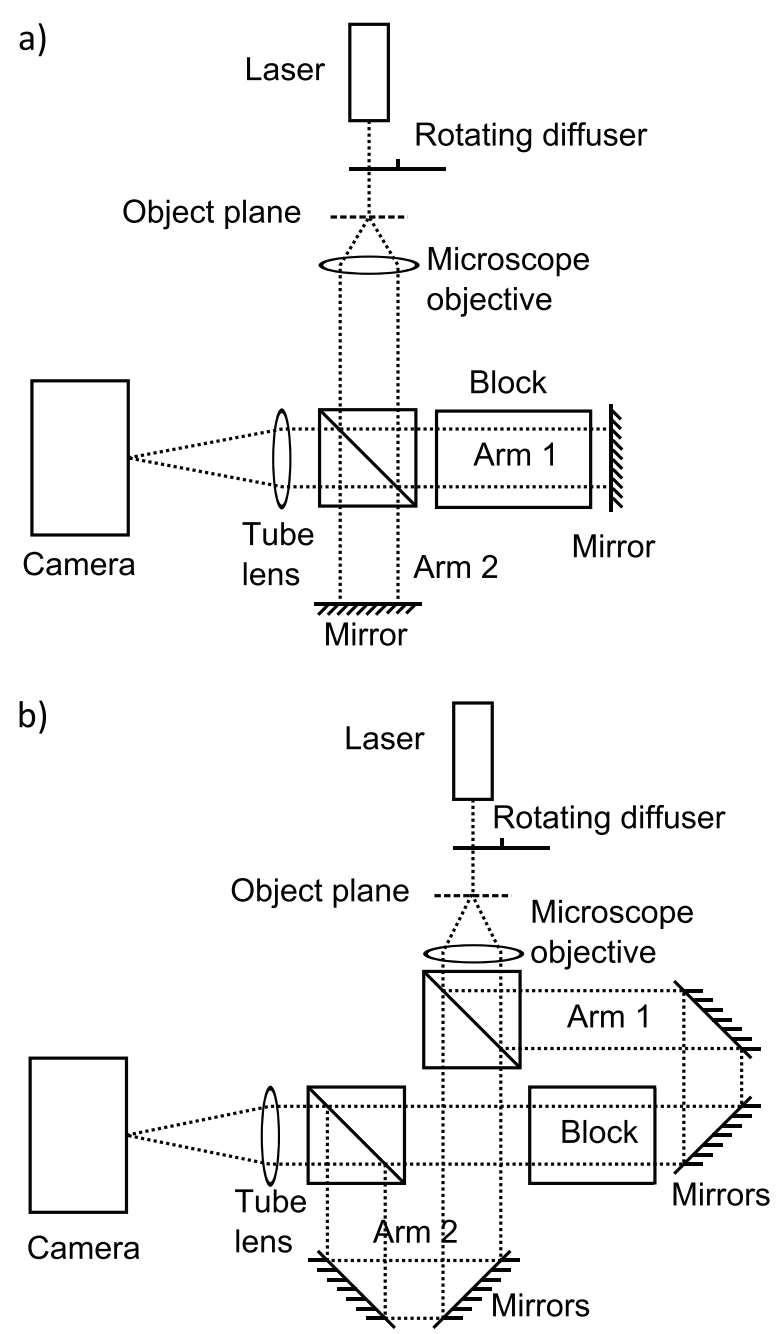

Figure 3. Schematic of the experimental arrangements used to demonstrate the principles. (a) Michelson interferometer configuration used to demonstrate infinity space balancing (section 3 ) and phase shifting (section 5). (b) Mach-Zehnder interferometer used to demonstrate carrier fringe generation, (section 4).

the two arms together with Snell's law and the trigonometric identity: $\sin ^{2} \theta+\cos ^{2} \theta=1$. For a typical configuration, such as the Mach-Zehnder interferometer used in [11], both paths will contain some glass components (e.g. beam splitter cubes or right-angle prisms) as well as air gaps, therefore both air and glass path in the two arms must be taken into account. Hence an expression for the OPD as a function of the bundle angle/image position can be written as:

$$
\mathrm{OPD}(\theta)=\frac{n_{a}\left(l_{1, a}-l_{2, a}\right)}{\cos \theta}+\frac{n_{g}^{2}\left(l_{1, g}-l_{2, g}\right)}{\sqrt{n_{g}^{2}-n_{a}^{2} \sin ^{2} \theta}}
$$

Where: $l_{1, a} l_{1, g}$ and $l_{2, a}, l_{2, g}$ are the geometric path lengths in the air and glass in the two arms; $n_{a}$ and $n_{g}$ are the refractive indices of the air and glass block respectively; and $\theta$ is the angle the bundle of rays (in air) from an object point traverse the infinity-space. In order to estimate the geometric path $l_{2, a}$ required to ensure a nearly constant OPD change across the image, equation (6) can be approximated by a Taylor series at $\theta=0$ giving: 
Table 1. Details of the demonstration interferometers.

\begin{tabular}{|c|c|c|}
\hline & $\begin{array}{l}\text { (a) Michelson } \\
\text { configuration }\end{array}$ & (b) Mach-Zehnder configuration \\
\hline Imaging system & \multirow{2}{*}{\multicolumn{2}{|c|}{$\begin{array}{l}\text { Olympus PlanApo } 1.25 \mathrm{x} \text { infinity corrected objective and tube lens. } \\
0.1 \mathrm{~mm} \text { grid slide illuminated by a rotating diffuser. }\end{array}$}} \\
\hline Object & & \\
\hline Arm 1, air path & $70.0 \mathrm{~mm}$ & $326.0 \mathrm{~mm}$ \\
\hline Arm 1, glass path ${ }^{\mathrm{a}}$ & $160.0 \mathrm{~mm}$ & $80.0 \mathrm{~mm}$ \\
\hline Total optical path, $l_{1}$ & $313.0 \mathrm{~mm}$ & $447.6 \mathrm{~mm}$ \\
\hline Apparent infinity space ${ }^{b}$ & $175.3 \mathrm{~mm}$ & $378.6 \mathrm{~mm}$ \\
\hline Arm 2, air path ${ }^{\mathrm{c}}$ & $175.3 \mathrm{~mm}$ & $378.6 \mathrm{~mm}$ \\
\hline Arm 2, glass path ${ }^{\mathrm{a}}$ & $0.0 \mathrm{~mm}$ & $0.0 \mathrm{~mm}$ \\
\hline Total optical path, 12 (=apparent infinity space) & $175.3 \mathrm{~mm}$ & $378.6 \mathrm{~mm}$ \\
\hline Optical path difference (OPD) & $137.9 \mathrm{~mm}$ & $69.0 \mathrm{~mm}$ \\
\hline
\end{tabular}

a Excluding paths common to both arms (e.g. inside beam splitters).

${ }^{\mathrm{b}}$ Calculated using equation (5).

${ }^{\mathrm{c}}$ Path where infinity space is balanced.

(a) $-10 \mathrm{~mm}$

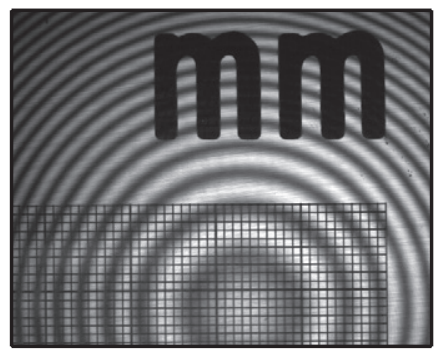

(b) Approaching balance

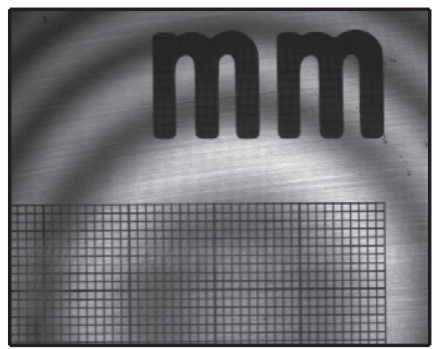

(c) $+10 \mathrm{~mm}$

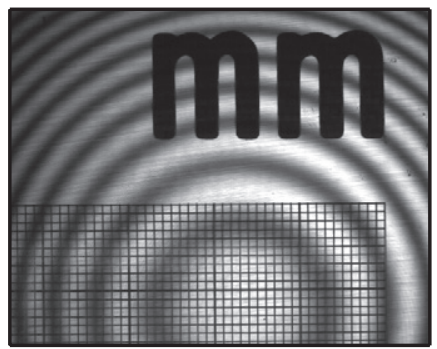

Figure 4. Examples of interferograms recorded using the Michelson interferometer described in table 1(a) with a $0.1 \mathrm{~mm}$ grid slide placed at the object plane. (a) Unbalanced infinity spaces approximately $-10 \mathrm{~mm}$ from balance; (b) approaching balanced infinity-space, $l_{2, \text { air }}=175.3 \mathrm{~mm}$, and (c) unbalanced infinity-spaces, approximately $+10 \mathrm{~mm}$ from balance. Here a portion of the grid and scale label are visible to demonstrate the image alignment.

$\mathrm{OPD}(\theta)=\mathrm{OPD}(\theta)+\left[\frac{\partial \mathrm{OPD}}{\partial \theta}\right]_{0} \cdot \theta+\left[\frac{\partial^{2} \mathrm{OPD}}{\partial \theta^{2}}\right]_{0} \cdot \frac{\theta^{2}}{2}+\ldots$

From this it can be seen that to minimise the variation across the image it is necessary to minimise the derivative terms, evaluating these at $\theta=0$ gives:

$$
\begin{gathered}
{\left[\frac{\partial \mathrm{OPD}}{\partial \theta}\right]_{0}=0} \\
{\left[\frac{\partial^{2} \mathrm{OPD}}{\partial \theta^{2}}\right]_{0}=n_{a}\left(l_{1, a}-l_{2, a}\right)+\frac{n_{a}^{2}\left(l_{1, g}-l_{2, g}\right)}{n_{g}}}
\end{gathered}
$$

The first derivative is equal to zero at $\theta=0$ due to symmetry about the optical axis, so setting the second derivative to zero to minimise equation (7) and rearranging leads to an expression for the required air path in the second arm:

$$
l_{2, a}=l_{1, a}+\frac{n_{a}}{n_{g}} l_{1, g}-\frac{n_{a}}{n_{g}} l_{2, g}
$$

This can also be considered to be a process of matching or balancing the apparent infinity-space lengths of the two arms with the block appearing to have a shorter infinity-space length as described in section 2. This balancing is analogous to the situation in a conventional imaging interferometer where the glass block length must be selected to match the magnification in the images produced by the two arms.

Finally, the additional OPD that will be added by including a glass block in one arm whilst maintaining the balancing of the infinity spaces is given by:

$$
\mathrm{OPD}=l_{b}\left(n_{g}-n_{a}\right)+l_{b}\left(\frac{1-n_{a}}{n_{g}}\right)
$$

Where there first term is the additional OPD due to the higher refractive index of the block, and the second term is the required additional air path in the arm to maintain the balancing of the infinity spaces.

To demonstrate this experimentally a Michelson interferometer, shown in figure 3(a), was constructed using a standard infinity corrected microscope objective and tube lens, with the details shown in table 1(a). According to equation (11) the $80 \mathrm{~mm}$ glass block included in one arm of the interferometer will add $\sim 69 \mathrm{~mm}$ of OPD per pass, when the infinity spaces are balanced. Here $l_{2, a}$ can be calculated by applying equation (10) or by calculating the apparent infinity-space lengths of the two arms (also included in table 1) via equation (5) and adding the necessary air path. Experimental interferograms are shown in figure 4 for the unbalanced cases figures 4 (a) and (c), when $l_{2, a}$ is varied by 
(a) Optical path, $l_{1}$

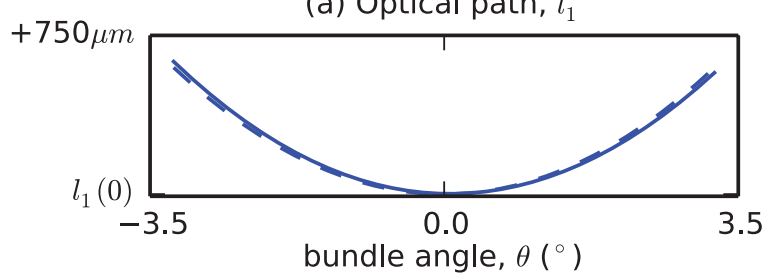

(b) Optical path, $l_{2}$

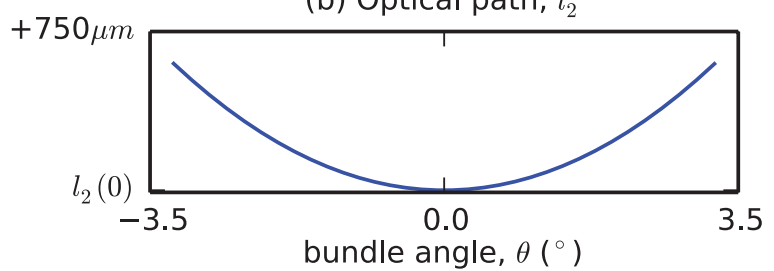

(c) Optical path difference

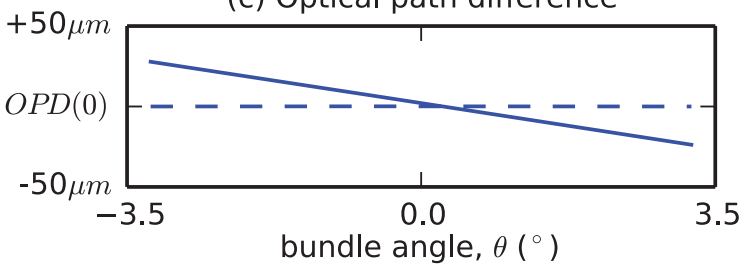

(d) Normalised fringe intensity

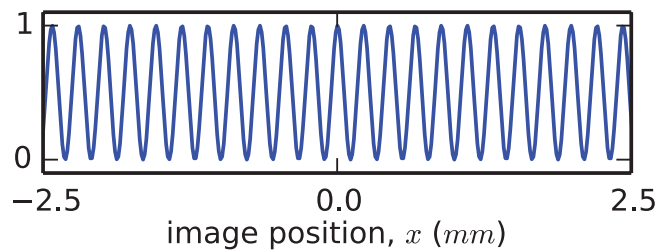

Figure 5. Numerical simulation of carrier fringe generation using a tilted glass block in single-pass configuration for the Mach-Zehnder interferometer described in table 1(a) Optical path length of the 1 st arm with an un-tilted (dashed) and tilted (solid) block, showing the slight offset of the minima. (b) Optical path length of the 2nd arm, (c) the resulting optical path differences no-tilt (dashed) tilted (solid) and (d) the resulting carrier fringe intensity.

approximately $\pm 10 \mathrm{~mm}$ either side of the balanced point and when approaching the balanced configuration, figure 4(b). When the balance point is reached the central fringe is 'fluffed' out to fill the entire image.

\section{Carrier fringe generation}

The introduction of linear carrier fringes and Fourier domain processing $[13,14]$ is a commonly used, single interferogram method of evaluating the interferometric phase $[5,15$, 16]. This section describes a simple and convenient means to introduce carrier fringes using a glass block placed in a single pass arrangement inside the infinity-space of one arm of the interferometer. The interferometer must first be configured to have approximately balanced infinity-spaces as described in section 3 then, by simply tilting the block with respect to the optical axis, carrier fringes can be generated.
The direction of the tilt controls the fringe direction and the magnitude of the tilt controls the fringe spatial frequency. This allows the introduction of linear carrier fringes with fine control over the magnitude and direction of the fringes, in a way which requires no other realignment of the interferometer, as opposed to the tilting of mirrors or prism within the interferometer [4] which results in an image offset which then needs to be corrected.

This principle behind this is shown in figure 5 which shows the results of numerically simulating the Mach-Zehnder configuration described in figure 3(b) and table 1 for a small block tilt of $0.5^{\circ}$. In figures 5(a) and (b) the optical path lengths of the two arms are shown plotted against bundle angles When the block is un-tilted the minima in optical path is symmetrical around zero (shown as the dashed curve) however when a tilt is applied to the block the bundle angle at which the minima in the optical path occurs is shifted (shown as the solid line). When the two paths are subtracted the resulting OPD, shown in figure 5(c) for the un-tilted (dashed line) and tilted cases (solid line), results in an approximately linear change with ray bundle angle and hence image position. This results in linear carrier fringes as shown in figure 5(d), the calculated fringe intensity.

This tilt can be introduced in each of the axial directions resulting in carrier fringes that can be aligned in any direction and changing the magnitude of the tilt will increase or decrease the magnitude of the OPD change in that direction. To demonstrate this experimentally, a Mach-Zehnder interferometer was constructed, as detailed in table 1(b), as this technique cannot be implemented in a Michelson interferometer configuration due to the requirement for a single pass through the block. Although the above analysis is only performed in one spatial dimension, the effect is the same for tilts applied in the other direction or a combination of the two, with the direction of the resulting fringes changing. Experimental examples of interferograms with carrier fringes introduced in this way are shown in figure 6 for various tilt directions and magnitudes. In this example, using a $80 \mathrm{~mm}$ block, typical tilts of around $0.5-2.5^{\circ}$ are required to produce spatial carrier fringes that can be resolved by the camera, however as the carrier fringe frequency is proportional to the block length used a longer block will require less tilt to produce the same result.

An expression relating the spatial carrier fringe frequency in the image plane, $\nu_{\mathrm{c}}$, to the tilt of the block can be found from the gradient of the OPD across the image. The OPD in this case, can be written similarly to equation (6) and including a new term for the optical path inside the tilted block.

$$
\begin{aligned}
\operatorname{OPD}(\theta)= & \frac{n_{a}\left(l_{1, a}-l_{2, a}\right)}{\cos \theta}+\frac{n_{g}{ }^{2}\left(l_{1, g}-l_{2, g}\right)}{\sqrt{n_{g}^{2}-n_{a}^{2} \sin ^{2} \theta}} \\
& +\frac{n_{b}{ }^{2} l_{b}}{\sqrt{n_{b}{ }^{2}-n_{a}{ }^{2} \sin ^{2}(\theta+\delta)}}
\end{aligned}
$$

Where, $\delta$ is the block tilt relative to the optical axis and $l_{b}$, $n_{b}$ are the block length and refractive index respectively. By 
(a) Vertical/Low frequency

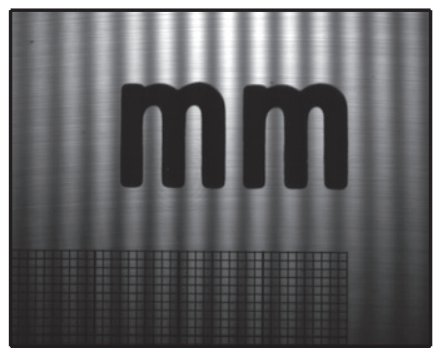

(b) Vertical/High frequency

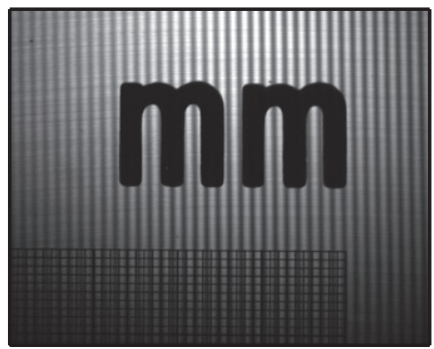

(c) Horizontal/Low frequency

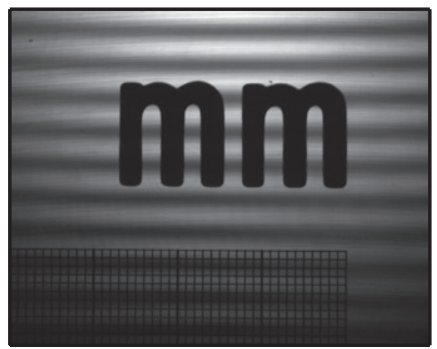

Figure 6. Examples of interferograms recorded using the Mach-Zehnder interferometer described in table 1(b). (a) vertical fringes generated by tilting the block about the vertical axis; (b) higher frequency fringes generated by increasing the magnitude of the tilt, and (c) horizontal fringes generated by rotating the blocking about the horizontal axis.

writing this in terms of image position, $x^{\prime}$, using equation (2), and assuming that the gradient is linear in $x^{\prime}$, i.e. the fringes have a constant frequency across the image, then the spatial carrier frequency can be found by evaluating this at the centre of the image, i.e. $x^{\prime}=0$ :

$$
\begin{aligned}
\nu_{\mathrm{c}} & =\frac{1}{\lambda}\left[\frac{\partial \mathrm{OPD}}{\partial x^{\prime}}\right]_{0} \\
& =\left(\frac{l_{b}}{f_{2} \lambda}\right)\left(\frac{n_{a}{ }^{2} n_{b}{ }^{2} \sin \delta \cos \delta}{\left(n_{b}{ }^{2}-n_{a}{ }^{2} \sin ^{2} \delta\right)^{3 / 2}}\right)
\end{aligned}
$$

Here $\lambda$ is the illuminating wavelength and $f_{2}$ is the focal length of the second lens. Finally this relationship can be simplified using a 1 st order Taylor series to given an expression for the block tilt required for a given fringe spatial frequency:

$$
\delta=\left(\frac{f_{2} \lambda}{l_{b}}\right)\left(\frac{n_{b}}{n_{a}^{2}}\right) \nu_{\mathrm{c}}
$$

\section{Phase shifting}

A related effect can be shown for the case of a tilted glass block in a double-pass configuration such as in a Michelson interferometer. In this case the tilt of the block will no longer produce carrier fringes as the shift in the minima of the optical path for each pass through the block is now in opposite directions and hence cancels, and the result is only an increase in the optical path in that arm, and hence a phase shift in the interferogram. This is shown in figure 7 which shows the results of numerically simulating the Michelson interferometer configuration described in figure 3(a) and table 1 for a small block tilt of $\sim 0.2^{\circ}$ which corresponds to approximately $2 \pi$ phase shift. In figure 7 (a) the optical paths versus bundle angle are shown for the 1st arm of the interferometer without tilt (shown by the dashed line) and with tilt applied (shown by the solid line). Here the increase in the optical path in this arm can be seen. Figure 7(b) shows the optical path in the second arm and figure 7(c) shows the resulting OPD that is approximately constant as a function of bundle angle. The resulting variation in the phase shift across the image is shown in figure 7(d), and is nearly constant across the field of view, for this configuration the maximum variation is around of only $1 \mathrm{mrad} / 0.02 \%$ for a $2 \pi$ phase shift.

An expression for the phase shift can be found by writing an expression for the OPD, with separate terms for the two passes through the block, with the direction of the tilt reversed:

$$
\begin{aligned}
\operatorname{OPD}(\theta)= & \frac{n_{a}\left(l_{1, a}-l_{2, . a}\right)}{\cos \theta}+\frac{n_{g}{ }^{2}\left(l_{1, g}-l_{2, g}\right)}{\sqrt{n_{g}^{2}-n_{a}{ }^{2} \sin ^{2} \theta}} \\
& +\frac{n_{b}{ }^{2} l_{b}}{\sqrt{n_{b}{ }^{2}-n_{a}{ }^{2} \sin ^{2}(\theta+\delta)}} \\
& +\frac{n_{b}{ }^{2} l_{b}}{\sqrt{n_{b}{ }^{2}-n_{a}{ }^{2} \sin ^{2}(\theta-\delta)}}
\end{aligned}
$$

Where, $\delta$ is the block tilt relative to the optical axis and $l_{b}, n_{b}$ are the block length and refractive index respectively. The phase shift applied at a given bundle angle for the block tilt can then be found from:

$$
\Delta \phi(\theta, \delta)=\frac{2 \pi}{\lambda}[\mathrm{OPD}(\theta, \delta)-\operatorname{OPD}(\theta, \delta=0)]
$$

Which can be expanded to:

$$
\begin{aligned}
\Delta \phi(\theta, \delta)= & \frac{2 \pi}{\lambda} n_{b} l_{b}\left\{\left[1-\left(\frac{n_{a}}{n_{b}}\right)^{2} \sin ^{2}(\theta+\delta)\right]^{-1 / 2}\right. \\
& \left.+\left[1-\left(\frac{n_{a}}{n_{b}}\right)^{2} \sin ^{2}(\theta-\delta)\right]^{-1 / 2}+\left[1-\left(\frac{n_{a}}{n_{b}}\right)^{2} \sin ^{2}(\theta)\right]^{-1 / 2}\right\}
\end{aligned}
$$

This can be simplified further using a 2nd order Taylor series, as the phase shift is symmetric around $\theta=0$ the first order term is zero, to give an expression relating phase shift to block length and tilt at the centre of the image:

$$
\Delta \phi(\theta=0, \delta)=\frac{2 \pi}{\lambda}\left(\frac{n_{a}^{2}}{n_{b}}\right) l_{b} \delta^{2}
$$

A demonstration of this effect can be seen in the experimental interferograms, captured using the Michelson interferometer described in section 3, that are shown in figure 8 . Here an approximately $2 \pi$ phase shift has been applied to the circular fringe pattern by rotating the block. 
(a) Optical path, $l_{1}$

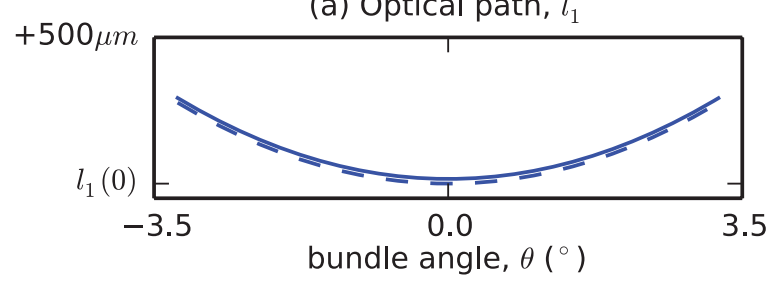

(b) Optical path, $l_{2}$

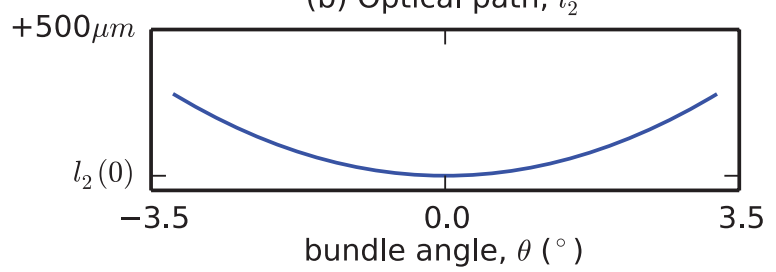

(c) Optical path difference

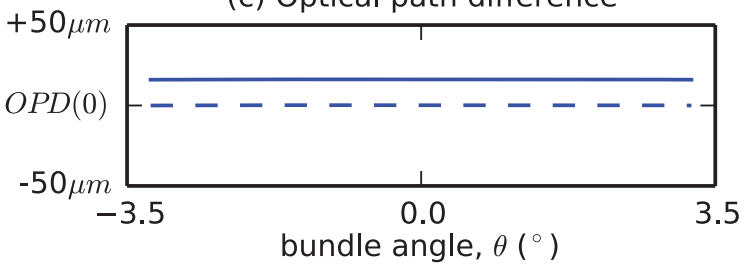

(d) Variation in phase shift

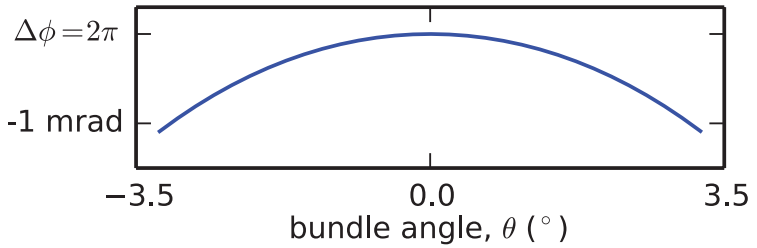

Figure 7. Numerical simulation of phase shifting using a tilted glass block in double-pass configuration for the Michelson interferometer described in table 1(a) optical path length of the 1st arm with un-tilted (dashed) and tilted (solid) block, (b) optical path length of the 2 nd arm, (c) the resulting optical path difference and (d) the variation in the phase shift as a percentage of the shift in the centre of the image. (a) Unshifted

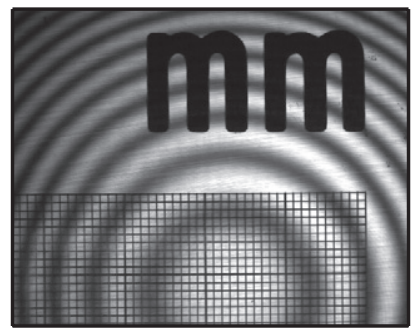

(b) Shifted $2 p i$

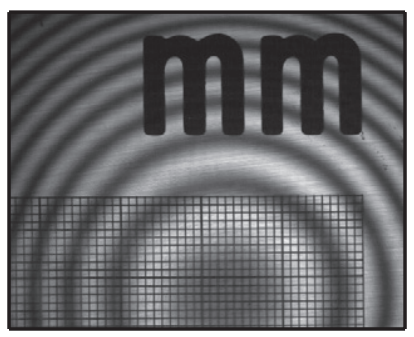

Figure 8. Examples of interferograms recorded using the Michelson interferometer described in table 1(a) with an approximately $2 \pi$ phase shift applied by tilting the glass block between the images shown in parts (a) and (b).

\section{Conclusions}

The theory and practical implementation of path-length imbalanced full-field interferometers constructed using infinity corrected optics has been described, with concepts introduced utilising glass blocks for the balancing of the infinity-spaces, and a convenient method for introducing spatial carrier fringes and phase shifting. Experimental interferograms demonstrating all three of these concepts have also been presented using Michelson and Mach-Zehnder interferometer configurations.

The concept of balancing of the infinity-spaces of the two arms (section 3) is critical to the implementation of pathlength imbalanced full-field interferometers to minimise the optical path difference variation across the image, and ensure adequate sampling of the fringes on the detector. This is especially important where large optical path differences, $\sim 360 \mathrm{~mm}[5,11]$, are required, such as in Doppler flow visualisations and quantitative measurements. Expressions have been derived for the required paths lengths in each arm to achieve this balance, equation (10), and the additional OPD that will be added by including a glass block of given length and refractive index in one arm whilst maintaining the balancing of the infinity spaces, equation (11).

A simple and convenient means to introduce carrier fringes using the tilt of a glass block placed in a single pass arrangement inside the infinity-space of one arm of the interferometer has been described in section 4 , where the direction of the tilt controls the fringe direction and the magnitude of the tilt controls the fringe spatial frequency. An expression relating the block tilt, length and refractive index, the tube lens focal length and the resulting spatial carrier frequency has been derived, equation (14).

Finally the use of a tilted glass block in a double-pass arrangement has been described for phase stepping, and expressions for the phase shift generated by a given block length, refractive index and tilt have been derived, equations (17) and (18). Numerical computations show that for a typical configuration the variation of the phase shift across the image is around $1 \mathrm{mrad} / 0.02 \%$ for a $2 \pi$ phase shift.

\section{Acknowledgments}

The authors acknowledge the support of the Engineering and Physical Sciences Research Council (EPSRC) UK, via grant $\mathrm{EP} / \mathrm{H} 02252 \mathrm{X} / 1$ and EP/G033900/1. Enquiries for access to the data referred to in this article should be directed to researchdata@cranfield.ac.uk.

\section{References}

[1] Abramowitz M, Spring K R, Long J C and Davidson M W 2015 Olympus microscopy primer: infinity-corrected optical systems, www.olympusmicro.com/primer/anatomy/ infinityhome.html

[2] Tkaczyk T S 2009 Field Guide to Microscopy vol FG13 (Bellingham, WA: SPIE Optical Engineering Press)

[3] Seiler F, Havermann M, George A, Leopold F and Srulijes J 2003 Planar velocity visualization in high-speed wedge flow using doppler picture velocimetry (DPV) compared with particle image velocimetry (PIV) J. Vis. $6253-62$

[4] Lu Z, Charrett T O H, Ford H D and Tatam R P 2007 Mach-Zehnder interferometric filter based planar Doppler velocimetry (MZI-PDV) J. Opt. A: Pure Appl. Opt. 9 1002-13 
[5] Lu Z, Charrett T O H and Tatam R P 2009 Three-component planar velocity measurements using Mach-Zehnder interferometric filter-based planar Doppler velocimetry (MZI-PDV) Meas. Sci. Technol. 20034019

[6] Pichler A, George A, Seiler F, Srulijes J and Havermann M 2009 Doppler picture velocimetry (DPV) applied to hypersonics Shock Waves SE: 80 ed K Hannemann and F Seiler (Berlin: Springer) pp 503-8

[7] Landolt A and Roesgen T 2009 Anomalous dispersion in atomic line filters applied for spatial frequency detection Appl. Opt. 48 5948-55

[8] Huang J, Ford H D and Tatam R P 1996 Phase-stepped speckle shearing interferometer by source wavelength modulation Opt. Lett. 21 1421-3

[9] Atcha H and Tatam R P 1994 Heterodyning of fibre optic electronic speckle pattern interferometers using laser diode wavelength modulation Meas. Sci. Technol. 5 704-9

[10] Francis D, James S W and Tatam R P 2007 Surface strain measurement using multi-component shearography with coherent fibre-optic imaging bundles Meas. Sci. Technol. 18 3583-91

[11] Charrett T O H, Bledowski I A, James S W and Tatam R P 2014 Frequency division multiplexing for interferometric planar Doppler velocimetry Appl. Opt. 53 4363-74

[12] Nobes D S, Ford H D and Tatam R P 2004 Instantaneous, three-component planar Doppler velocimetry using imaging fibre bundles Exp. Fluids 36 3-10

[13] Roddier C and Roddier F 1987 Interferogram analysis using Fourier transform techniques Appl. Opt. 26 1668-73

[14] Takeda M, Ina H and Kobayashi S 1982 Fourier-transform method of fringe-pattern analysis for computer-based topography and interferometry J. Opt. Soc. Am. 72 156-160

[15] Seiler F, Pichler A, Pfaff R and George A 2008 Improved Doppler picture velocimetry and new automated processing 14th Int. Symp. on Application of Laser Techniques to Fluids (Lisbon, Portugal) pp 07-3.6

[16] Landolt A and Roesgen T 2009 Global Doppler frequency shift detection with near-resonant interferometry Exp. Fluids 47 733-43 


\section{Cranfield University}

2015-12-01

Full-field interferometry using infinity corrected optics

Charrett, Thomas O. H.

IOP Publishing

TOH Charrett and R P Tatam, Full-field interferometry using infinity corrected optics,

Measurement Science and Technology, vol. 27, Iss. 1, pp. 015402, 2016, http://stacks.iop.org/0957-0233/27/i=1/a=0154 http://dx.doi.org/10.1088/0957-0233/27/1/015402

Downloaded from Cranfield Library Services E-Repository 